\title{
Zika, and rapid diagnostic tests for malaria
}

\author{
Fiona Godlee editor in chief
}

The BMJ

A year ago few people outside French Polynesia had heard of Zika. Now few people in the world will not have heard of it. This relatively benign and largely asymptomatic infection has spread to 25 countries, apparently bringing with it microcephaly, a condition now haunting thousands of families in-and travelling from-Latin America, the Pacific islands, and elsewhere (Cape Verde).

As Daniel Lucey explains in his editorial (doi:10.1136/bmj. i781), the guidance is clear and unanimous on what precautions people should take to prevent transmission to an unborn child. But the absence of an effective diagnostic test bedevils efforts to contain and respond to the virus.

The world is responding, though never quickly enough, says Lucey. The Ebola virus epidemic taught us many lessons, but "this time our response must be faster and smarter-to control the Zika epidemic, and in preparation for the many other infectious disease epidemics surely to come," he says.

The BMJ and other journals are doing what we can to support research and provide reliable and up to date information for health workers in affected areas. We have pulled together relevant resources from across the BMJ publishing house and other sources (bmj.com/freezikaresources). In concert with the other members of the International Committee of Medical Journal Editors (icmje.org), we are making all Zika related research freely available and waiving publication fees. And The $B M J$ has joined other organisations such as the Wellcome Trust and the Bill and Melinda Gates Foundation in an initiative to share data on Zika (www.wellcome.ac.uk/News/Media-office/ Press-releases/2016/WTP060169.htm).
With Zika understandably dominating the headlines, other mosquito-borne diseases have, of course, not gone away. Prevalence of the world's most burdensome of these, malaria, is falling in many countries, say Eleanor Ochodo and colleagues (doi:10.1136/bmj.i107). But with this success come other challenges, notably the harms caused by presumptive overtreatment of fever as malaria.

The policy of presumptive treatment made sense when the prevalence of malaria was high and antimalarials were cheap, and it dominated the approach to fever in malarial regions of the world. Now the ingrained belief among health workers that missing a case of malaria is more dangerous than treating a fever as malaria is fuelling an epidemic of its own, with adverse effects of drugs, rising costs, and resistance to the drugs.

Despite the development and scaling up of rapid diagnostic tests for malaria, presumptive overtreatment of fever as malaria still goes on: a test may not be done for various reasons; the test may deliver a false positive result; or antimalarials may still be prescribed despite a negative test.

Ochodo and colleagues outline strategies to tackle these problems. But they conclude that changing behaviour is rarely easy and that malaria experts will need to learn from efforts to tackle antibiotic resistance in other illnesses.

For all The BMSs latest articles on the Zika virus epidemic go to bmj. co/zika.

Cite this as: BMJ 2016;352:i841

๑ BMJ Publishing Group Ltd 2016 